\title{
captadores a cuerda vibrante: su fundamento y aplicaciones
}

ALBERTO FUENTES, Lcdo. en Ciencias Físicas División de Metrología del Instituto Eduardo Torroja

Sinopisis La reciente adquisición por el Instituto Eduardo Torroja de un equipo de medida para captadores a cuerda vibrante nos ha sugerido la publicación del presente artículo, en el cual se resumen la teoría y aplicaciones de tales captadores, cuyo uso se está extendiendo mucho en los últimos años, no sólo en las grandes obras como presas y puentes, sino también en los laboratorios de la construcción.

\section{Fundannemto de los captradomes}

La teoría de vibraciones (Refs. 1 y 2) expresa que una cuerda tensa sujeta en dos puntos $A$ y $B$ (fig. 1), distantes $l$ y sometida a una tensión $T$, cuando oscila libremente lo hace con una frecuencia $f$ dada por la expresión:

$$
f=K \frac{\sqrt{T}}{l}
$$

donde $K$ es una constante cuyo valor depende de las características físicas de la cuerda. La relación existente entre la frecuencia de oscilación de la cuerda y la tensión a que está sometida permite que toda magnitud que influya sobre la tensión $T$ pueda, en principio, ser medida a tra. vés del conocimiento de la citada frecuencia.

Captadores que realizan el acoplamiento entre la variable a medir y la tensión a que se somete a una cuerda

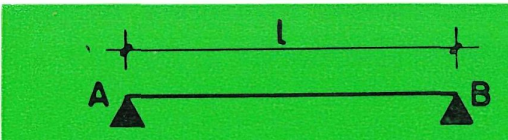

Fig. 1

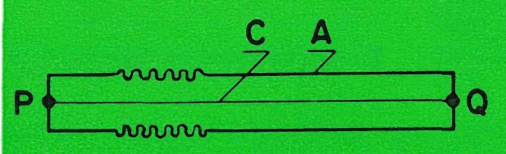

Fig. 2 - elemento sensible del captador-han sido realizados para diversas magnitudes, explicándose, a continuación, de forma esquemática, el fundamento de algunos de ellos (*).

Captadores para medida de deformaciones (figs. 2, 3 y 4).

El cilindro $A$, deformable longitudinalmente, contiene la cuerda $C$, tensa, fijada a sus extremos $P$ y $Q$. Cualquier alargamiento o acortamiento del cilindro se transmite a la cuerda, variando así su tensión y, con ello, la frecuencia de su oscilación. La figura 3 muestra un cap-

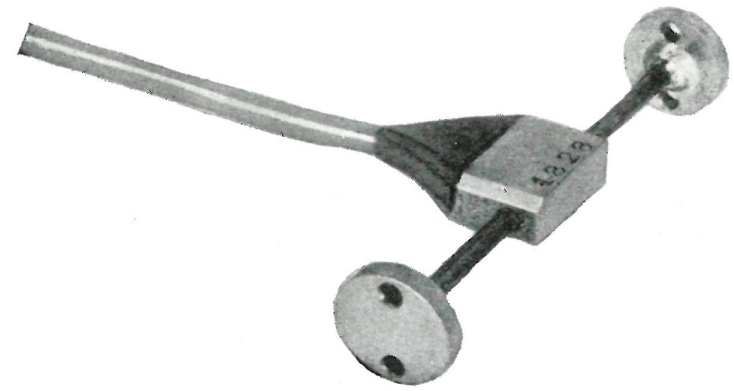

Fig. 3 tador para medidas en el interior de estructuras de hormigón. Se introduce en el mismo en el momento del hormigonado, y el captador empotrado así, en él, sigue sus deformaciones. La figura 4 corresponde a otro captador para medidas en la superficie de estructuras de hormigón, acero, etc. El captador es fijado a la estructura mediante las abrazaderas que se ven en los extremos del mismo.

Captadores para medida de presión (figs. 5 y 6).

(*) Indiquemos que las cuerdas utilizadas consisten en un hilo de acero. Este hilo trabaja en la zona elástica, lo que hace aplicable la fórmula [1]. Asimismo es realizado en acero de gran calidad, lo que, unido a la estanquidad del captador, le proporciona una gran estabilidad en el tiempo. 


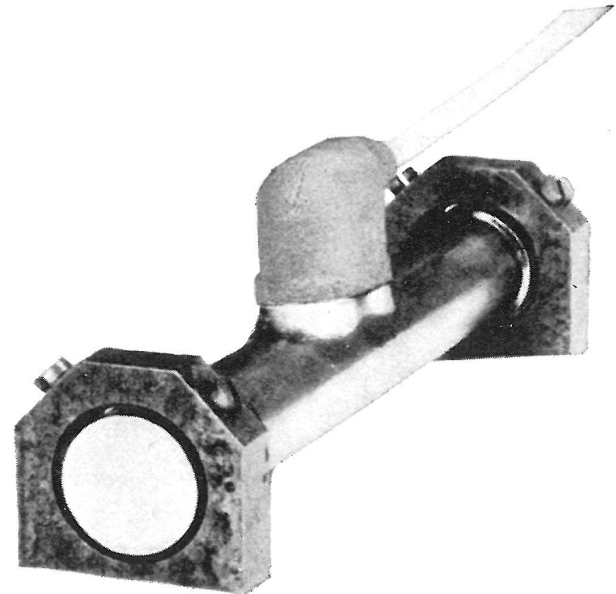

Fig. 4
En el cilindro de la figura la presión aplicada sobre $A$ se traduce en un acortamiento de la dimensión $h$, cambiando así la tensión a que está sometida la cuerda $C$ y, por tanto, la frecuencia de su vibración. La figura 6 corresponde a un tipo comercial de captador de presión.

Clinómetros (figs. 7 y 8).

El clinómetro consiste, en esencia, en el péndulo representado en la figura 7. Cuando la placa $A$ deja de estar horizontal, la masa $M$ flecta al vástigo $B$ cambiando la longitud de la cuerda $C$ y, con ello, la frecuencia de su vibración. La figura 8 presenta un tipo comercial de clinómetro.

Mediante distintas versiones de los montajes esquematizados se puede, en fin, medir otras magnitudes, existiendo en la actualidad captadores a cuerda vibrante para la medida de deformaciones, presiones, fuerzas de compresión y de tensión, presión de aguas intersticiales, temperatura, etc.

\section{Esquemen gemeral del circuito de medide}

Una vez indicado cómo trabajan los captadores que estudiamos, el problema que queda por resolver es el de la determinación de la frecuencia de vibración de la cuerda del captador. Naturalmente tendremos que efectuar antes la excitación de dicha vibración. Esto podemos

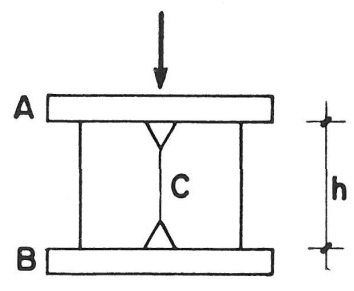

Fig. 5

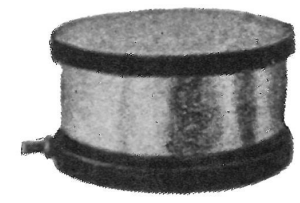

Fig. 6

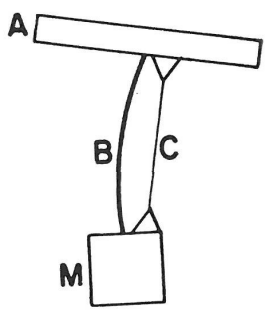

Fig. 7

conseguirlo fácilmente aprovechando el circuito de la figura 9. Si cerramos instantáneamente el interruptor $A$, un impulso de corriente atravesará la bobina $B$, creando un campo magnético que ejercerá una fuerza sobre el hilo de acero $\left(^{*}\right)$, sacándolo así de su posición de reposo. Puesto que la acción del impulso es instantánea, la cuerda comienza a vibrar libremente con la frecuencia dada por la ecuación [1]. Tal oscilación se atenúa inevitablemente en un con la frecuencia dada por la ecuación [1]. Tal oscilación se atenúa inevitablemente en un corto intervalo de tiempo, obligándonos a hacer la medida de su frecuencia en ese breve espacio de tiempo o a pulsar varias veces el interruptor $A$ hasta que logremos realizarla. Para evitar este inconveniente algunos equipos sostienen la vibración de la cuerda mediante un

(*) Si se usa un hilo de acero es, precisamente, para que a causa de las propiedades magnéticas de este material sufra los efectos de los campos magnéticos. 


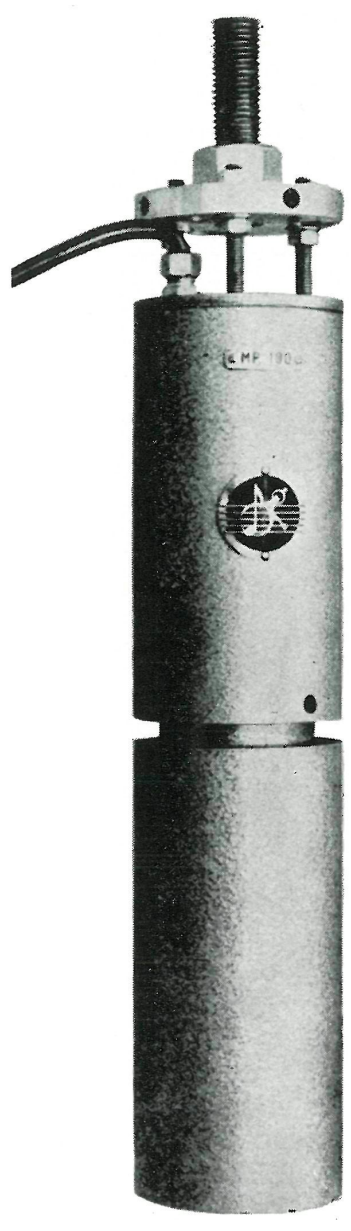

Fig. 8

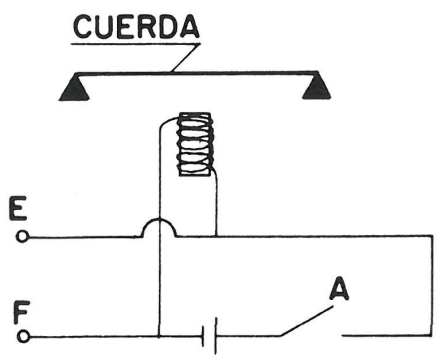

Fig. 9 oscilador, diseñado de forma tal que oscile a la frecuencia de libre vibración de la misma. Precisamente la cuerda es el elemento determinante de la frecuencia de oscilación del oscilador. En la figura 10 se esquematiza

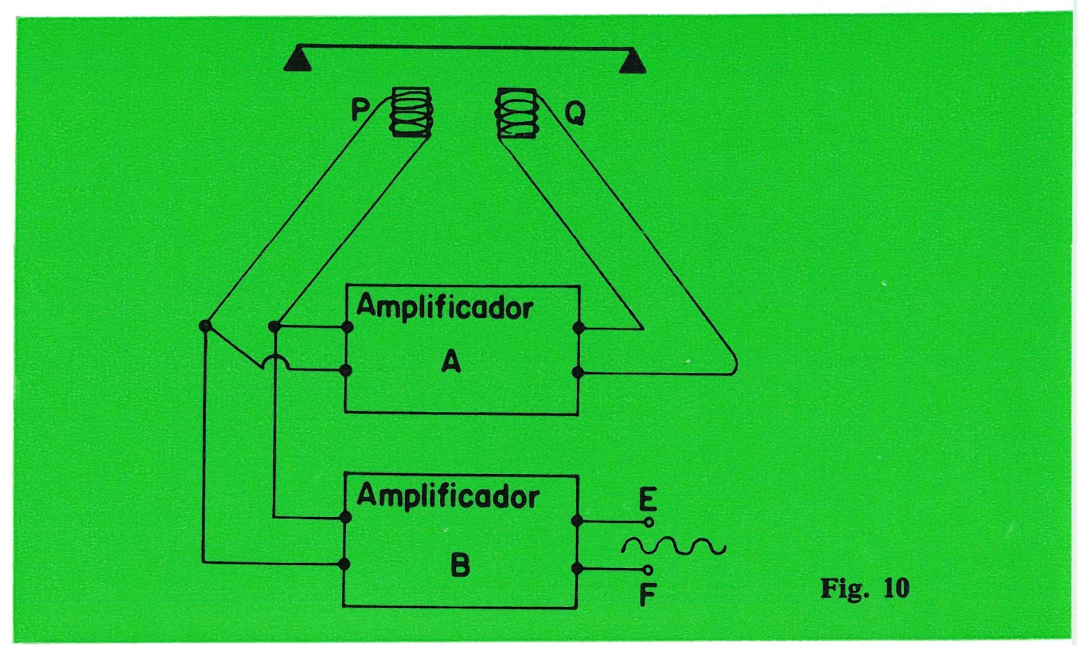

un oscilador de este tipo. La vibración de la cuerda induce una señal eléctrica de igual frecuencia en la bobina $P$, la cual, convenientemente amplificada y defasada en el amplificador $A$, sostiene la oscilación a través de la bobina $Q$. El punto de trabajo del amplificador es aquel en el que sólo la frecuencia de resonancia-la de libre oscilación de la cuerda-está presente. Finalmente, la señal producida por la bobina $P$, amplificada en el amplificador $B$, es utilizada para la medida de la frecuencia de la vibración.

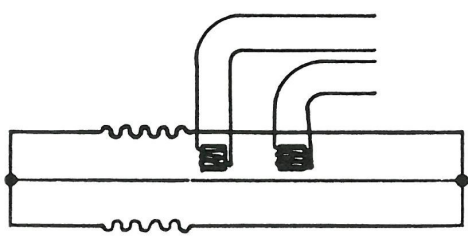

Fig. 11

De lo dicho se desprende que las bobinas $P$ y $Q$ citadas han de estar próximas a la cuerda, de forma que los captadores esquematizados en el apartado 1 llevan como partes integrantes del mismo dos bobinas. El captador para medida de deformaciones de la figura 2 será entonces tal como se le representa en la figura 11.

Pensemos ahora en la manera de realizar la medida de la frecuencia de la señal que aparece entre las terminales $E$ y $F$ de la figura $10\left({ }^{*}\right)$. (*) Si se tratase de un equipo en el que no se sostiene la oscilación de la cuerda, habría de medirse la
frecuencia de la señal que durante un corto intervalo de tiempo aparece entre los terminales $E$ y $F$ de la figura 9. 


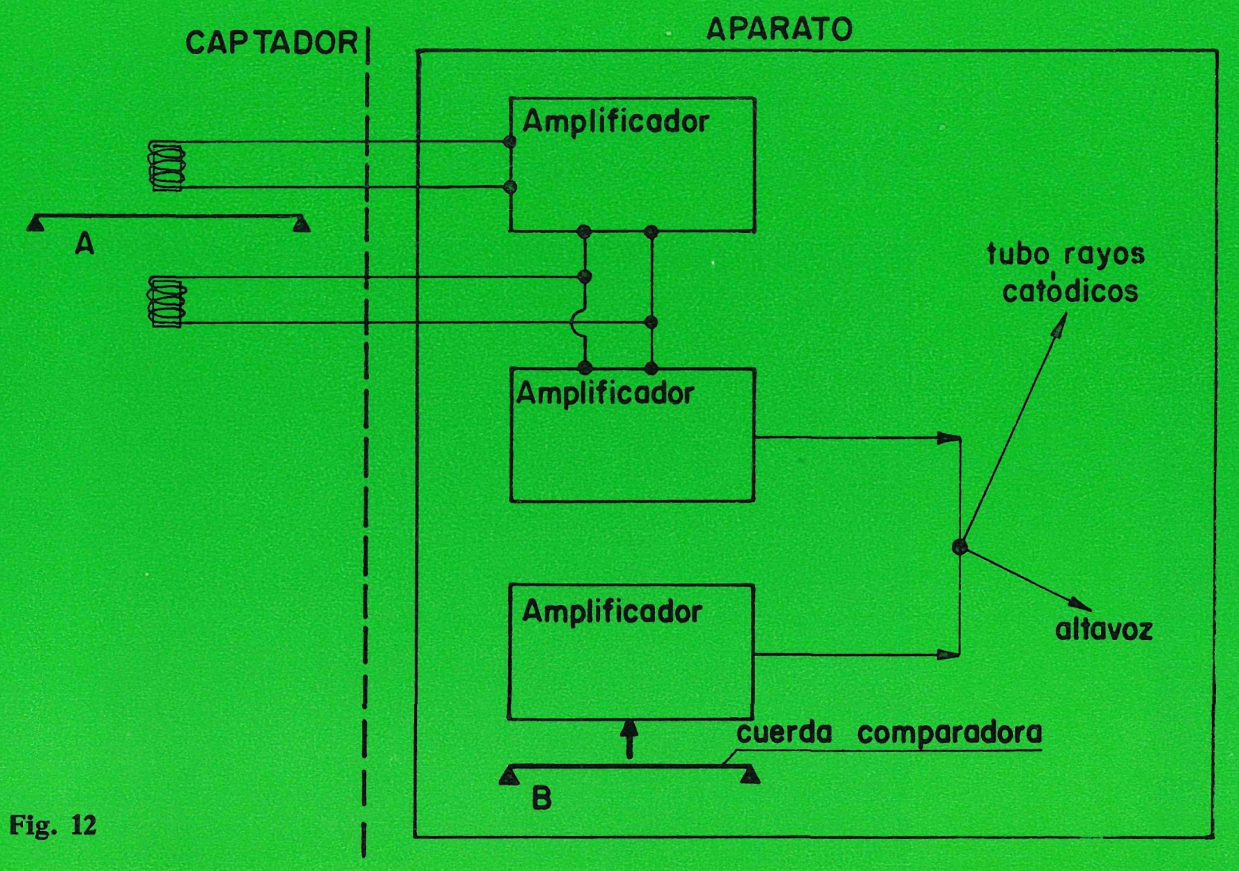

Normalmente los equipos comerciales comparan la frecuencia a medir con la de oscilación de otra cuerda, calibrada $(*)$, insertada en el equipo de medida, a la que se le varía la tensión hasta que vibre con igual frecuencia que la del captador. Las señales provenientes de las dos cuerdas son enviadas a un tubo de rayos catódicos o un altavoz en los que la condición de igualdad de frecuencias es fácilmente detectada. El esquema de bloques completo de un equipo tal sería el dado en la figura 12. El dial que gobierna la tensión a que está sometida la cuerda $B$ de comparación está graduado normalmente en microdeformaciones, de manera que esta magnitud puede ser leída directamente. La figura 13 representa un equipo comercial que responde, en esencia, al esquema de la figura 12.

Naturalmente, cualquier otro método de medir la frecuencia es válido. Pueden ser utilizados frecuencímetros y entre ellos los digitales, es decir, aquellos que expresan numéricamente, según algún código, el valor de la frecuencia. El esquema de un equipo es el que puede verse en la figura 14. Una medida de este tipo, medida digital, tiene todo el interés de la presentación digital de datos: fidelidad, posibilidad de introducir los datos directamente en calculadora, etc. (véase Ref. 3). La figura 15 representa un equipo digital para 50 canales.

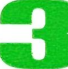

\section{Camatemisticas de las medialas mealizudus nediante coptradores a cuerda vilbram tre y aplicoriomes de éstos}

La característica más importante que presentan las medidas realizadas mediante captadores a cuerda vibrante nace del hecho de que la magnitud a medir-deformación, fuer-

(*) Indiquemos que esta comparación con una cuerda idéntica a la del captador, calibrada directamente en la magnitud a medir, nos libra de los errores que se derivarían de aplicar la fórmula [1] no exacta (véase Ref. 2).

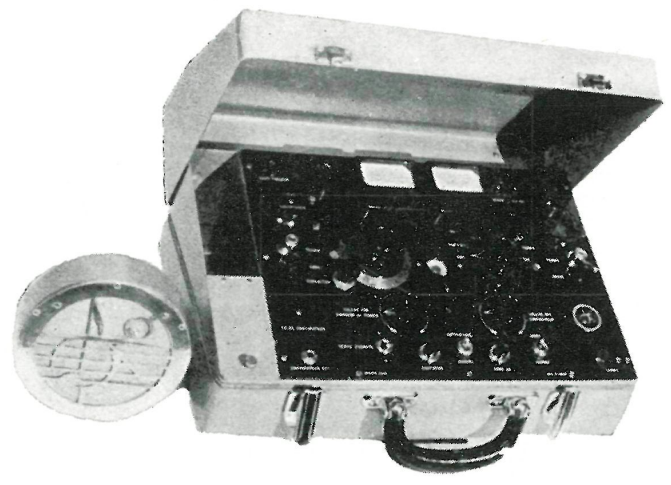

Fig. 13 


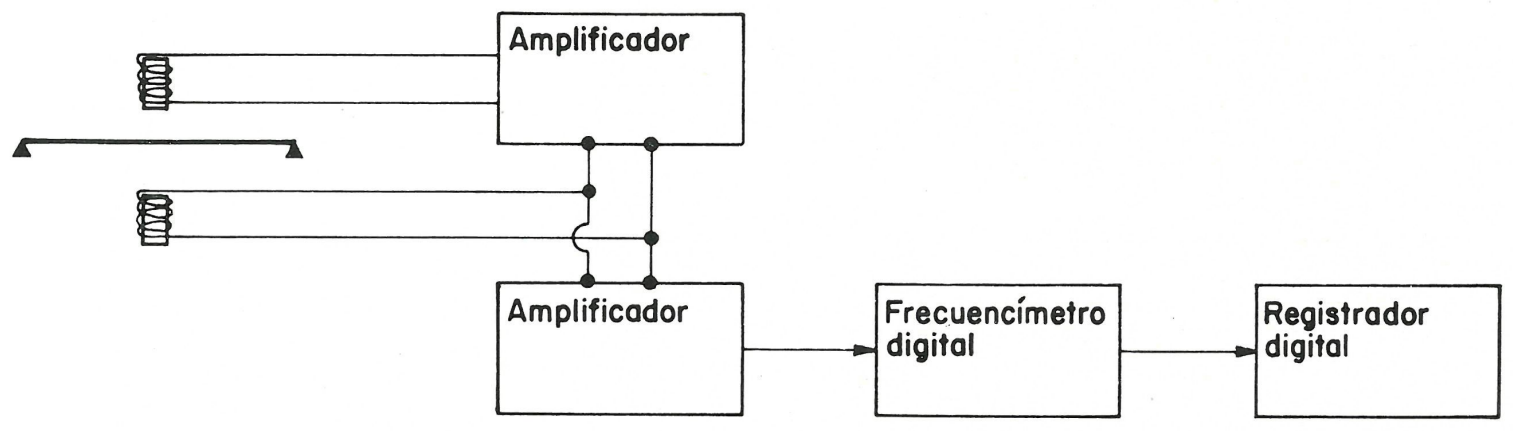

Fig. 14

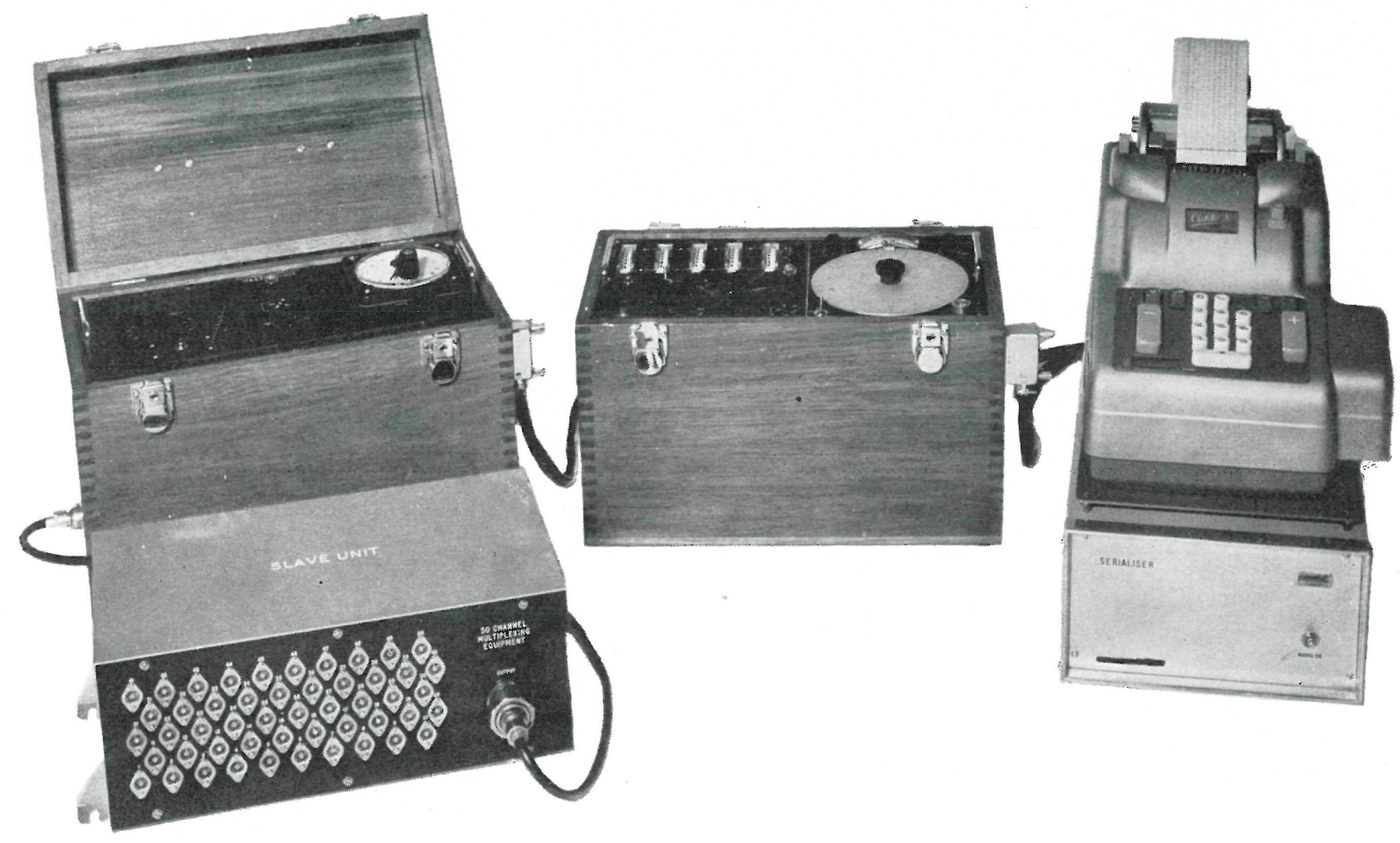

Fig. 15

za, temperatura, etc.- -es traducida a una oscilación eléctrica de una determinada frecuencia. Cuando un captador produce como señal un voltaje $\left(^{*}\right)$, tal señal es alterada inevitablemente por las resistencias y capacidades de fuga a masa que presenta la línea que enlaza el captador con el aparato de medida (fig. 16) y por la propia impedancia de esa línea. A causa de ello, la señal que llega al aparato de medida es distinta de la producida por el captador, introduciéndose de esta forma un error en la medida.

(*) Muchos captadores dan como señal una variación de resistencia, pero ésta para ser medida es convertida a la postre en un voltaje. 


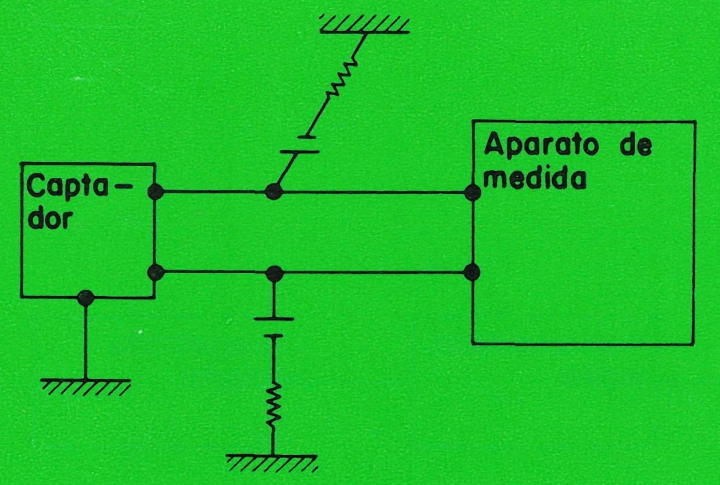

Fig. 16

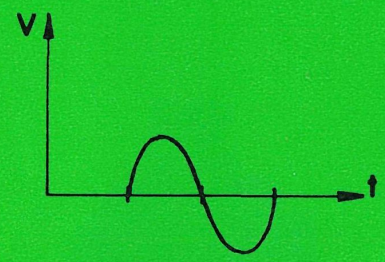

Salida del captador

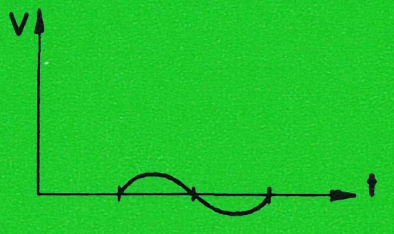

Entrada al aparato de medido

Fig. 17 etcétera, pues resisten bien la humedad y los golpes que puedan sufrir durante su colocación en tales ambientes. Estas características, y la fidelidad en la transmisión de frecuencias, con independencia de la impedancia de la línea y sus variaciones, da a estos captadores una gran estabilidad en el tiempo. Por fin, el hecho, acabado de citar, referente a la fidelidad en la transmisión, permite largas líneas de enlace, existiendo la posibilidad de que haya grandes distancias entre los puntos de medida y el lugar donde la medida ha sido hecha.

Todo lo dicho justifica que los captadores a cuerda vibrante, aparte de su uso junto con otros tipos de captadores en medidas de laboratorio, sean los elementos ideales para realizar medidas en presas, puentes, fundaciones, etc., y que tales medidas puedan ser seguidas durante largos períodos de tiempo. En la referencia 4 pueden verse interesantes ejemplos de la aplicación de estos captadores, así como un estudio teórico completo del fundamento de los mismos.

\section{Referemcius}

1. Coulson: «Ondas» (Editorial Dossat).

2. E. Torroja y J. A. Petrirena: «Sobre los errores de la medida de las deformaciones en el interior de los macizos por aplicación de la teoría de las cuerdas vibrantes». (Asoc. Esp. para el Prog. de las Ciencias. XIV Congreso, 1934).

3. A. Fuentes: «Esquema general de las medidas eléctricas» (Monografía del I.E.T.c.c.).

4. J. M. Tobío: «Ensayos no destructivos» (pendiente de publicación). 


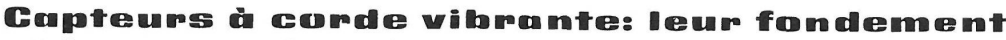 et leurs applicentions}

Alberto Fuentes, Licencié ès Sciences Physiques

La récente acquisition par l'Institut Eduardo Torroja d'un équipement de mesure pour capteurs à corde vibrante nous a suggéré la publication de cet article qui résume la théorie et les applications de ces capteurs, dont l'emploi s'est étendu considérablement au cours de ces dernières années, non seulement pour les grands ouvrages tels que des barrages et des ponts, mais aussi pour les laboratoires de la construction.

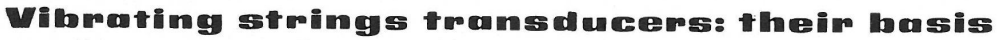 and applications}

Alberto Fuentes, Physics graduate

The present article has been inspired by the recent introduction, at the Instituto Eduardo Torroja, of a vibrating string transducer. In this article the theory and applications of these instruments is set forth. Their use is becoming very extensive in recent years, not only at the large projects, such as dams and bridges, but also at construction laboratories.

\section{Erschleicher mit Schwingsalutht. Seine Frumallage und Amwerndumg}

Alberto Fuentes, Dipl.-Physiker

Der neue kauf vom Institut Eduardo Torroja von einer Messausrüstung für Erschleicher mit Schwingsdraht ist den Grund von der Veröffentlichung vom vorliegenden Atikel, in dem die Theorie und Anwendungen zusammengefasst sind. Seine Anwendung ist in den letzten Jahren grösser geworden. Man benutzt ihm nicht nur in den grossen Bauten wie Staustufen und Brücken sondern auch in den Baulaboratorien. 